\title{
Dinâmica de nitrogênio e fósforo em reservatório na região semiárida utilizando balanço de massa
}

\section{Ticiana F. Vidal ${ }^{1} \&$ José Capelo Neto ${ }^{2}$}

${ }^{1}$ UFC. Fortaleza, CE. E-mail: ticianafvidal@yahoo.com.br (Autor correspondente)

${ }^{2}$ UFC/DEHA. Fortaleza, CE. E-mail: capelo@ufc.br

\section{Palavras-chave:}

macronutrientes

eutrofização

qualidade de água

\begin{abstract}
R E S U M O
Muitos reservatórios são alvo de impactos resultantes de diversas atividades antrópicas, principalmente os localizados nos domínios das bacias metropolitanas. O açude Gavião, responsável pelo abastecimento de água potável da Região Metropolitana de Fortaleza, apresenta a presença excessiva de fitoplâncton, consequência direta do enriquecimento da água por nutrientes, dificultando substancialmente o tratamento da água no reservatório e elevando os custos de tratamento. Torna-se imprescindível, portanto, estudos, planos de gerenciamento, ações corretivas e/ou preventivas a fim de que, futuramente, a qualidade das águas não seja prejudicada a ponto de culminar na necessidade da modificação da tecnologia de tratamento utilizada na estação. A determinação da carga de nutrientes e os balanços de massa podem avaliar o processo de eutrofização no sistema verificando-se se há exportação ou retenção de nutrientes no corpo d'água. Amostras mensais de janeiro a maio de 2011 foram coletadas em cinco pontos do açude com o objetivo de estudar o balanço de massa dos macronutrientes. No período de estudo o açude acumulou cerca de 28,06 t de fósforo e 121,42 $\mathrm{t}$ de nitrogênio podendo ser classificado eutrófico.
\end{abstract}

Key words: macronutrients eutrophication water quality

\section{Dynamics of nitrogen and phosphorus in reservoir in the semi-arid region using mass balance}

\begin{abstract}
A B S T R A C T
Many reservoirs are subjected to impacts resulting from various anthropogenic activities, especially those located in metropolitan areas of the basins. The reservoir Gavião is responsible for drinking water supply in the Metropolitan Region of Fortaleza and contains excessive presence of algae, a direct result of the enrichment of water by nutrients, which substantially hampers the treatment of water collected in the reservoir and increase the treatment costs. Therefore, it is essential to carry out studies of corrective and/or preventative management plans so that future water quality is not impaired to the point that it culminates with the replacement or modification of the technology used in the treatment plant. The determination of nutrient loading and mass balances can evaluate the eutrophication process in the system, checking for export or retention of nutrients in the water body. Samples were collected monthly from January to May 2011, at five points in the reservoir in order to study the mass balance of macronutrients. During the study period the reservoir accumulated approximately $28.06 \mathrm{t}$ of phosphorus and $121.42 \mathrm{t}$ of nitrogen, and can be classified as eutrophic.
\end{abstract}

\section{INTRODUÇÃo}

A falta de um planejamento sustentável durante o processo de ocupação do solo, intensificada no Brasil a partir da década de 60, acarretou sérios impactos à sociedade como um todo, a exemplo da contaminação dos recursos hídricos constituindo uma forte ameaça à saúde pública (Oliveira et al., 2009).

Segundo Duarte \& Vieira (2009), muitos impactos negativos são gerados pela utilização dos meios hídricos que funcionam como receptores de descargas pontuais e difusas, responsáveis pela eutrofização progressiva dos corpos hídricos e consequente alteração da sua estrutura trófica. Em função da eutrofização muitos reservatórios e lagos no mundo já perderam a capacidade de abastecimento humano, de manutenção da vida aquática e de recreação. Um número crescente de trabalhos vem sendo desenvolvido demonstrando a gravidade do problema (Sampaio et al., 2002; Bennion et al., 2005; Pinto-Coelho et al., 2005).

A eutrofização das águas significa seu enriquecimento por nutrientes, sobretudo nitrogênio e fósforo, levando ao crescimento excessivo das plantas aquáticas. Um dos principais impasses relacionados à eutrofização é a proliferação de cianobactérias em detrimento de outras espécies aquáticas. Quando submetidas a determinadas condições ambientais muitos gêneros de cianobactérias podem produzir toxinas que chegam a ser fatais aos animais e aos seres humanos (Conley et al., 2009; Rabalais et al., 2009). 
No Ceará o monitoramento da água dos açudes que são mananciais de abastecimento humano, como o açude Gavião, vem apresentando elevadas concentrações de nitrogênio e fósforo, constituindo fator de preocupação por parte do poder público e das populações usuárias das águas (Figueirêdo et al., 2007).

O monitoramento da água, seja no aspecto quantitativo ou qualitativo, fundamenta a tomada de decisões e previne conflitos entre usuários evidenciados especialmente no semiárido nordestino cujas características ambientais do meio físico geram problemas bastante peculiares (Souza Filho et al., 2006; Gorayeb et al., 2006; Machado et al., 2012).

A área de estudo, isto é, o açude Gavião, encontra-se posicionada na região nordeste do estado do Ceará, inserido no município de Pacatuba e faz parte do Sistema Integrado de Abastecimento da Região Metropolitana de Fortaleza.

Ressalta-se que o açude Gavião está localizado à montante da Estação de Tratamento de Água - ETA, responsável pelo abastecimento de água potável da Região Metropolitana de Fortaleza. Águas eutrofizadas proporcionam maior dificuldade e elevação nos custos de tratamento. A presença excessiva de fitoplâncton afeta substancialmente o tratamento da água captada no reservatório devido à necessidade de: remoção do fitoplâncton, remoção de cor, remoção de sabor e odor, maior consumo de produtos químicos e lavagens mais frequentes dos filtros, entre outros; desta forma, é imprescindível que açudes com tendência a sofrer processos de eutrofização sejam alvo de estudos, monitoramentos, planos de gerenciamento, ações corretivas e/ou preventivas para que, futuramente, a qualidade de suas águas não seja prejudicada a ponto de culminar na substituição/modificação da tecnologia utilizada atualmente nas estações de tratamento.

A partir daí constata-se a importância de se observar e estudar a dinâmica dos nutrientes presentes no açude Gavião. O objetivo do trabalho é estudar o balanço de massa dos macronutrientes no açude Gavião dando ênfase aos compostos nitrogenados e fosfatados, determinar as cargas relacionando a concentração das espécies químicas com a vazão de entrada e saída do açude podendo, desta forma, determinar o balanço de massa desses materiais e inferir sobre a capacidade do açude de reter ou exportar nutrientes e ainda investigar o nutriente limitante do açude a partir da relação entre nitrogênio e fósforo.

\section{MATERIAl E MÉtodos}

Amostras de água foram coletadas no açude Gavião em 5 pontos distintos ao longo de seis campanhas, com periodicidade mensal entre janeiro e maio de 2011. As amostragens foram realizadas no período da manhã, entre 9 e $12 \mathrm{~h}$; os pontos escolhidos foram: GAV05, GAV14, GAV16, Canal RiachãoGavião (Canal) e Riacho Gavião (Riacho), de acordo com a Figura 1; a escolha de cada ponto monitorado foi feita levandose em consideração a relevância estratégica de cada um em particular e sua localização dentro da bacia hidráulica.

A vazão de entrada do Riacho Gavião nos dois primeiros monitoramentos foi nula em razão do período de estiagem não

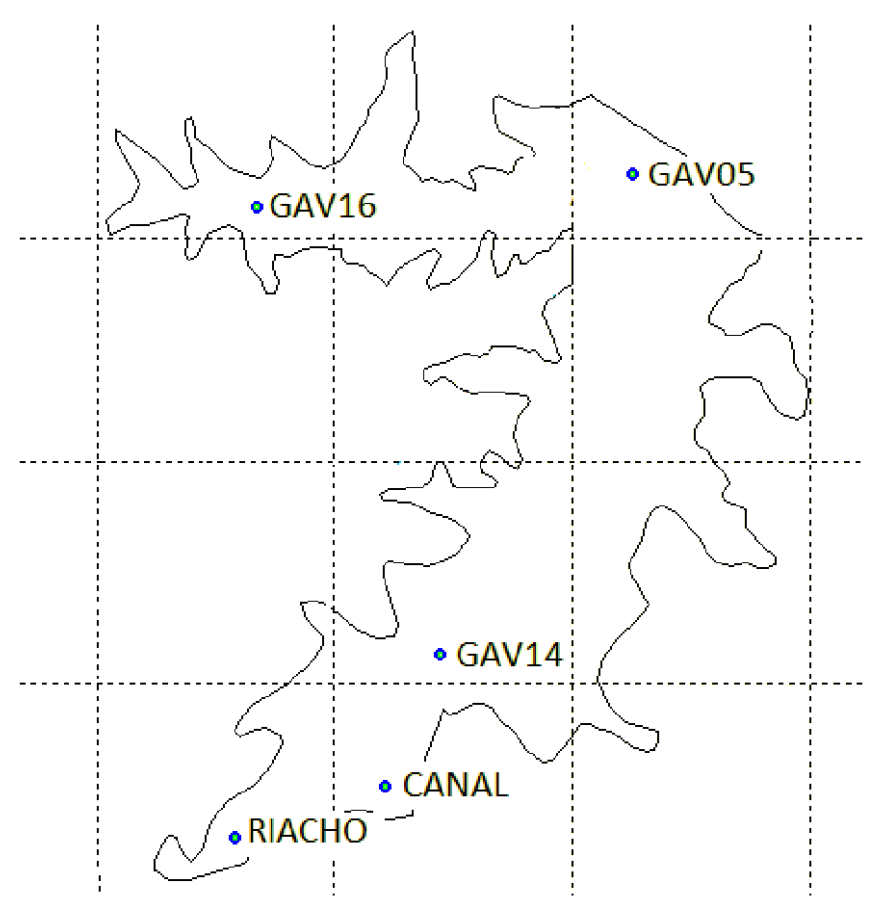

Fonte: GPS TrackMaker

Figura 1. Pontos de amostragem do açude Gavião

havendo, portanto, transferência de água para o açude através deste afluente. Com o início das chuvas de maior intensidade a partir do mês de março, o Riacho Gavião começou a alimentar o açude de maneira mais significativa e, portanto, passou-se a monitorar o ponto Riacho; já o ponto Canal foi monitorado durante todo o trabalho de vez que o mesmo alimentou o açude durante o período de estudo.

As amostras de água para análises das séries de nitrogênio (nitrato, nitrito, amônia e nitrogênio orgânico) e de Fósforo (fósforo total e ortofosfato) foram coletadas com amostrador de Van Dorn, imediatamente transferidas para frascos de vidro do tipo âmbar e mantidas a aproximadamente $4{ }^{\circ} \mathrm{C}$. Amostras para determinação de nutrientes dissolvidos foram filtradas em membranas de $0,45 \mu \mathrm{m}$ e analisadas dentro de um limite máximo de $48 \mathrm{~h}$ obedecendo sempre ao prazo de cada análise, conforme preconiza cada método. Todas as análises tiveram como referência Standard methods for the examination of water and wastewater (APHA, 2005) e foram realizadas em triplicata.

No ponto GAV05, localizado na região mais profunda do açude com aproximadamente $12 \mathrm{~m}$ de profundidade, foram coletadas amostras em 5 profundidades distintas: superfície $(0,3 \mathrm{~m})$, fundo $(0,5 \mathrm{~m}$ acima do sedimento) e três outras intermediárias (em torno de 3,6 e $9 \mathrm{~m}$ ), com o intuito de se obter mais informações do perfil do ponto considerado o mais representativo do açude.

Nos pontos GAV14 e GAV16 foram coletadas amostras em três profundidades dispostas da seguinte maneira: superfície, fundo e uma amostra intermediária (em torno de $3 \mathrm{~m}$, já que a profundidade máxima nestes dois pontos varia em torno de $7 \mathrm{~m}$ ). Os pontos que representam as entradas de água no reservatório - Canal e Riacho - apresentam profundidade média 
em torno de $4 \mathrm{~m}$. Neste caso, foram coletadas águas apenas na superfície.

O açude Gavião apresenta dois pontos principais de entrada que alimentam o reservatório ao longo do ano, são eles: o Canal Riachão-Gavião (Canal) e o Riacho Gavião (Riacho). Considerou-se, então, que a afluência total ao açude ocorreu principalmente pela soma das vazões desses dois afluentes.

Basicamente, a vazão do Canal é regulada para manter alimentada a ETA-Gavião, não variando significantemente durante o ano mantendo-se entre 6,9 e 8,5 $\mathrm{m}^{3} \mathrm{~s}^{-1}$ (COGERH, 2011).

Considerou-se, a partir de então, que nos períodos nos quais não há contribuição do Riacho, a vazão de entrada do açude é aproximadamente sua vazão saída, que no caso é a vazão de entrada da ETA, somada à evaporação.

A vazão de afluência ao açude através do Riacho é difícil de ser determinada já que ocorre durante poucos meses, além de ainda não se dispor de dados da morfometria do riacho motivo por que a vazão afluente ao açude Gavião foi estimada a partir de um balanço hídrico, no qual se consideraram a alteração no volume armazenado do açude (obtido a partir das leituras diárias das cotas), as taxas de evaporação, o vertimento e a vazão liberada pela barragem.

Dados de cota-volume do açude Gavião foram usados a fim de se determinar a vazão total de entrada no açude (Vazão do Canal + Vazão do Riacho). Utilizou-se a seguinte equação matemática para estimar a vazão (Vollenweider, 2000):

$$
V_{t+\Delta t}=V_{t}+I-E_{t}-S_{t}-R_{t}
$$

em que:

$\mathrm{V}_{\mathrm{t}+\Delta \mathrm{t}}$ - volume no tempo atual, $\mathrm{L}^{3}$

$\mathrm{V}_{\mathrm{t}}$ - volume no tempo anterior, $\mathrm{L}^{3}$

I - afluência total ao açude no tempo $\Delta \mathrm{t}, \mathrm{L}^{3} \mathrm{~T}^{-1}$

$\mathrm{E}_{\mathrm{t}} \quad$ - evaporação no açude no tempo $\Delta \mathrm{t}, \mathrm{L}^{3} \mathrm{~T}^{-1}$

$\mathrm{S}_{\mathrm{t}} \quad$ - vertimento, $\mathrm{L}^{3} \mathrm{~T}^{-1}$

$\mathrm{R}_{\mathrm{t}} \quad$ - retirada, $\mathrm{L}^{3} \mathrm{~T}^{-1}$

Rearranjando a Eq. 1 acima, tem-se:

$$
I=V_{t+\Delta t}-V_{t}+E_{t}+S_{t}+R_{t}
$$

Considerando a variação de volume $=\mathrm{V}_{\mathrm{t}+\Delta \mathrm{t}}-\mathrm{V}_{\mathrm{t}}=\Delta \mathrm{V}$, obtém-se:

$$
\mathrm{I}=\Delta \mathrm{V}+\mathrm{E}_{\mathrm{t}}+\mathrm{S}_{\mathrm{t}}+\mathrm{R}_{\mathrm{t}}
$$

A Eq. 3 rege a afluência total podendo ser utilizada para qualquer reservatório; no caso, quando aplicada ao açude Gavião, seu resultado expressa a soma das afluências do Riacho e Canal; a forma como cada termo da equação do balanço hídrico foi calculado é apresentada a seguir:

- O termo variação de volume $(\Delta \mathrm{V})$ foi obtido a partir da tabela Cota X Volume durante o período estudado $\left(\mathrm{m}^{3} \mathrm{~d}^{-1}\right)$;

- Os valores de evaporação $\left(E_{t}\right)$ foram baseados nos dados médios mensais obtidos de 2000 a 2010 no açude Gavião;
- O termo vertimento no açude $\left(\mathrm{S}_{\mathrm{t}}\right)$ foi estimado a partir de fórmula empírica com base na cota máxima de vertimento de $36,0 \mathrm{~m}$ :

Se cota $<36,0 \mathrm{~m}$, o vertimento foi nulo $(\mathrm{S}=0)$;

Se cota $>36,0$, utilizou-se a fórmula de Poncelet e Lesbros:

$$
\mathrm{S}=\mathrm{C} \cdot \mathrm{L}\left(\mathrm{H}-\mathrm{H}_{\mathrm{vert}}\right)^{1,5}
$$

em que:

C - coeficiente de vertimento (para vertedores retangulares $=1,77$ )

L - largura do vertedouro $(L=20,0 \mathrm{~m})$

$\mathrm{H}$ - cota atual

$\mathrm{H}_{\text {vert }}$ - cota de vertimento $\left(\mathrm{H}_{\text {vert }}=36,0 \mathrm{~m}\right)$

Com os valores obtidos e calculados realizou-se o balanço hídrico do reservatório; a partir dele, determinou-se uma estimativa da vazão afluente total ao açude Gavião durante o período estudado. Os valores médios mensais calculados durante o período são apresentados na Tabela 1 .

Tabela 1. Valores médios mensais da vazão afluente ao açude

\begin{tabular}{cc}
\hline Campanha & Afluência mensal $\left(\mathbf{m}^{\mathbf{3}} \mathbf{s}^{-1}\right)$ \\
Jan & 8,20 \\
Fev & 7,30 \\
Mar & 8,23 \\
Abr & 8,49 \\
Mai & 7,95 \\
\hline
\end{tabular}

O balanço de nutrientes foi realizado através da obtenção dos fluxos de entrada e saída de nutrientes no açude; as cargas foram calculadas a partir das concentrações de nutrientes e das vazões de água que entram e saem; Visando à elaboração do balanço de massa, algumas considerações foram feitas:

- A concentração de nitrogênio total (nitrito + nitrato + amônia $+\mathrm{N}_{\text {Orgânico }}$ ) foi usada no balanço de nitrogênio;

- O balanço para o fósforo foi calculado a partir do fósforo total;

- A concentração média de nutrientes em cada estrato foi calculada levando-se em consideração uma área de influência para cada ponto amostral, de acordo com as distâncias entre cada ponto; a determinação desta área de influência foi uma tentativa de se atribuir pesos diferentes aos pontos de coleta (Eq. 5). De maneira geral:

$$
\mathrm{CR}=\frac{\left(\mathrm{C}_{\mathrm{GAV} 05} \times \mathrm{P}_{05}\right)+\left(\mathrm{C}_{\mathrm{GAV} 14} \times \mathrm{P}_{14}\right)+\left(\mathrm{C}_{\mathrm{GAV} 16} \times \mathrm{P}_{16}\right)}{\left(\mathrm{P}_{05}+\mathrm{P}_{14}+\mathrm{P}_{16}\right)}
$$

em que:

CR - concentração de nutriente em cada estrato do reservatório em cada mês

$\mathrm{C}_{\mathrm{GAV} 05}$ - concentração no ponto GAV05

$\mathrm{P}_{05}$ - peso do ponto GAV05, $\mathrm{P}_{05}=2778,43$

$\mathrm{C}_{\mathrm{GAV} 14}$ - concentração no ponto GAV14

$\mathrm{P}_{14}$ - peso do ponto GAV14, $\mathrm{P}_{14}=1379,58$ 
$\mathrm{C}_{\mathrm{GAV} 16}$ - concentração no ponto GAV16

$\mathrm{P}_{16}$ - peso do ponto GAV16, $\mathrm{P}_{16}=1259,74$

A massa de nutrientes presente em cada estrato do reservatório, mês a mês, foi calculada multiplicando-se o volume de cada estrato (Tabela 2) pela concentração média obtida a partir da Eq. 5 cuja massa total foi obtida pela soma das massas de cada estrato.

Tabela 2. Cota-área-volume e estratos considerados

\begin{tabular}{|c|c|c|c|c|}
\hline \multirow{2}{*}{ Estratos } & Prof. & Cota & \multirow{2}{*}{$\begin{array}{l}\text { Área } \\
\left(\mathrm{km}^{2}\right)\end{array}$} & \multirow{2}{*}{$\begin{array}{c}\text { Volume } \\
\left(\mathrm{m}^{3}\right)\end{array}$} \\
\hline & \multicolumn{2}{|c|}{ (m) } & & \\
\hline \multirow{5}{*}{1} & 0 & 35,5 & 5,820 & 29520000 \\
\hline & 0,5 & 35 & 5,630 & 26660000 \\
\hline & 1,5 & 34 & 5,160 & 21230000 \\
\hline & 2,5 & 33 & 4,480 & 16400000 \\
\hline & 3,0 & 33,5 & 4,100 & 14350000 \\
\hline \multirow{4}{*}{2} & 3,5 & 32 & 3,720 & 12300000 \\
\hline & 4,5 & 31 & 3,010 & 8940000 \\
\hline & 5,5 & 30 & 2,430 & 6230000 \\
\hline & 6,0 & 30,5 & 2,170 & 5150000 \\
\hline \multirow{4}{*}{3} & 6,5 & 29 & 1,900 & 4070000 \\
\hline & 7,5 & 28 & 1,390 & 2430000 \\
\hline & 8,5 & 27 & 0,980 & 1260000 \\
\hline & 9,0 & 27,5 & 0,755 & 880000 \\
\hline \multirow{3}{*}{4} & 9,5 & 26 & 0,530 & 500000 \\
\hline & 10,5 & 25 & 0,240 & 120000 \\
\hline & 11,5 & 24 & 0 & 0 \\
\hline
\end{tabular}

O carregamento dos nutrientes ao açude foi calculado a partir da multiplicação da vazão calculada pela concentração em cada ponto (Riacho e Canal).

A saída de nutrientes pela ETA foi calculada levando-se em consideração a média ponderada das concentrações ao longo da coluna d'água no ponto GAV05 multiplicada pela vazão da ETA-Gavião.

O balanço de massa foi feito individualmente para cada mês de trabalho, possibilitando observar, mês a mês, as variações dos nutrientes contidos na massa líquida, a massa sedimentada ou desnitrificada, além das cargas de entrada e de saída.

Foram levadas em consideração apenas entradas e saídas no meio líquido. A equação seguinte foi utilizada a fim de se obter o balanço de massa para os nutrientes modificado de (Vollenweider, 2000):

$$
\mathrm{B}=\mathrm{C}_{\mathrm{A}}-\mathrm{R}
$$

em que:

B - balanço de nutrientes no mês

$\mathrm{C}_{\mathrm{A}}$ - massa de nutrientes que chega pelo Riacho ou Canal ao reservatório no mês

$\mathrm{R}$ - massa de nutrientes que sai do reservatório pela ETAGavião no mês

É válido ressaltar que o termo "B" pode ser positivo (retenção de nutriente) quanto negativo (exportação de nutriente) em dado período.

Para a elaboração do balanço foi adotada a cota máxima de referência $35,5 \mathrm{~m}$ já que na maioria do período de estudo o açude permaneceu em média nesta cota. Informações técnicas importantes, tais como a cota, a área e o volume do açude, foram obtidas a partir da Tabela 2, com vista à construção do balanço dos nutrientes.

Os estratos utilizados no balanço foram considerados de acordo com a Tabela 2 mostrando a cota de referência adotada $(35,5 \mathrm{~m})$ e as demais cotas até a profundidade máxima amostrada no açude (aprox. 11,5 m), com suas respectivas áreas em $\mathrm{km}^{2}$ e volumes em $\mathrm{m}^{3}$.

Considerou-se que o estrato 1 compreende da superfície até os $3 \mathrm{~m}$, o estrato 2 dos 3 até $6 \mathrm{~m}$, o estrato 3 representa de 6 até 9 $\mathrm{m}$ e o estrato 4 dos $9 \mathrm{~m}$ até próximo ao sedimento. É necessário ressaltar que apenas o ponto GAV05 possui os 4 estratos já que apresenta $12 \mathrm{~m}$ de profundidade, aproximadamente enquanto os pontos GAV14 e GAV16 apresentam em média $6 \mathrm{~m}$ de profundidade, sendo representados pelos estratos 1 e 2 e como os dois afluentes foram amostrados apenas na superfície, os mesmos contribuem apenas para o estrato 1 .

\section{Resultados e Discussão}

A Tabela 3 sinaliza a carga de entrada, saída e acúmulo de fósforo total que ocorreram no açude Gavião para cada mês. Pode-se observar que todos os balanços foram positivos sugerindo que este corpo d'água reteve cerca de 28,06 toneladas de fósforo durante o período estudado.

Para os fluxos de entrada, os valores ficaram entre $8,817 \mathrm{t}$ (fevereiro) e 15,877 t (abril) de fósforo aumentando ao longo do período estudado. Este comportamento era esperado já que, com o aumento das vazões afluentes, sobremaneira a do Riacho Gavião, o fluxo de nutrientes aumentou; para os fluxos de saída os valores oscilaram entre 5,868 e 8,204 t de fósforo.

As massas de fósforo total retidas variaram entre $0,613 \mathrm{t}$ no mês de fevereiro e 8,696 t no mês de março; neste caso ocorreu, em geral, tendência à diminuição da carga de saída ao longo do tempo indicando que em associação com o incremento da carga afluente houve um aumento da retenção de fósforo, representado pelo aumento do balanço até níveis de 8,696 mês $^{-1}$ nos períodos com maior precipitação (março, abril e maio).

Vários fatores físico-químicos podem interferir na imobilização dos íons fosfato no ambiente aquático, como a concentração dos íons de ferro, alumínio, sulfeto, compostos orgânicos e carbonatos, $\mathrm{pH}$ e condições de oxirredução (Freire et al., 2013). Uma explicação plausível para esta elevada retenção de fósforo no açude seria a precipitação do fósforo. Em lagos

Tabela 3. Fluxos de entradas, saída e balanços para o fósforo total em cada mês

\begin{tabular}{crcc}
\hline Mês & Entrada & Saída & Balanço \\
\cline { 2 - 4 } Jan & 9,259 & $\begin{array}{c}\text { (t mês } \\
\text { - })\end{array}$ & \\
Fev & 8,436 & 1,823 \\
Mar & 14,817 & 8,204 & 0,613 \\
Abr & 15,877 & 6,209 & 8,696 \\
Mai & 14,355 & 7,436 & 8,441 \\
\hline
\end{tabular}


onde predominam condições de oxidação grande parte dos íons de ferro presentes se encontra na forma oxidada $\left(\mathrm{Fe}^{3+}\right)$. As argilas, principais constituintes dos minerais secundários, também apresentam grande capacidade de adsorção de fosfato podendo haver ligação entre a carga negativa do fosfato com a positiva das bordas das argilas ou substituição do fosfato por silicatos da argila (Esteves, 2011).

Hijo (2009) verificou que o balanço entre os fluxos de entrada e saída no açude Castanhão indicou uma retenção de $135 \mathrm{t}$ de fosfato $\left(\mathrm{PO}_{4}^{3-}\right)$ durante o período chuvoso amostrado. No caso deste estudo observou-se um acúmulo de 28,06 t para o parâmetro com o método utilizado.

De acordo com a Tabela 4, a relação entre as concentrações e as vazões de entrada indicou fluxos de nitrogênio total (soma de todas as frações nitrogenadas quantificadas: nitrato, nitrito, amônia e nitrogênio orgânico), variando entre 21,082 (fevereiro) e 95,949 $\mathrm{t}$ mês $^{-1}$ (abril), com média de 57,893 $\mathrm{t}$ mês $^{-1}$. A carga de saída do açude apresentou variações entre 16,156 (janeiro) e $46,402 \mathrm{t}$ mês $^{-1}$ (também em abril). Verificou-se, para o balanço de fevereiro, único mês no qual o balanço ficou negativo (fluxo de saída superior ao fluxo de aporte), houve exportação de 3,939 $\mathrm{t}$ de nitrogênio, podendo o açude ter exportado tanto a massa efluente quanto na forma de gás.

Nos demais meses o balanço apresentou-se positivo indicando provável retenção do macronutriente no açude (sedimentação ou desnitrificação), resultando numa soma de 121,409 t de nitrogênio. Em geral, os índices de retenção ficaram maiores quando a precipitação na bacia se elevou visto que as cargas nas entradas se tornaram ainda maiores com o aporte de nutriente às águas do açude Gavião.

O balanço para o nitrogênio é mais susceptível a erros de vez que perdas positivas podem ocorrer devido à sedimentação tal como pelo balanço da nitrificação-desnitrificação. Valores negativos podem ser resultantes de carreamento interno ou ressuspensão de sedimentos.

Outra discussão relevante nos processos de eutrofização diz respeito à relação nitrogênio e fósforo totais caso em que se pretendeu calcular esta relação de modo a se estabelecer o fator limitante no processo de eutrofização.

$\mathrm{O}$ nitrogênio e o fósforo podem tornar-se nutrientes limitantes dos corpos d'água já que são consumidos pelo fitoplâncton em uma relação média N:P de 10:1 (Green \& Finlay, 2010; Correll, 1999). É importante notar que mesmo em cenários no qual o nitrogênio é fator limitante, há grandes possibilidades de a produção primária permanecer elevada uma vez que este nutriente é muito abundante na atmosfera podendo ser fixado pelo fitoplâncton (Xu et al., 2010).

Tabela 4. Fluxos de entradas, saída e balanços para o nitrogênio total em cada mês

\begin{tabular}{cccc}
\hline Mês & Entrada & Saída & Balanço \\
\cline { 2 - 4 } Jan & & (t mês $^{-1}$ ) & \\
Fev & 21,086 & 16,156 & 4,930 \\
Mar & 21,082 & 25,021 & $-3,939$ \\
Abr & 88,105 & 45,013 & 43,093 \\
Mai & 95,949 & 46,402 & 49,548 \\
\hline
\end{tabular}

A razão N/P foi calculada tomando-se as frações totais de nitrogênio e fósforo para cada mês de estudo e para cada porção do reservatório: interior do açude, entradas e saída; as concentrações médias no interior do açude foram calculadas usando-se a mesma média ponderada utilizada para o balanço de massa.

A Tabela 5 mostra as respectivas relações $\mathrm{N} / \mathrm{P}$ em cada mês individualmente, para os pontos localizados no interior do açude (média das concentrações em GAV05, GAV14 e GAV16), para as zonas de afluência (médias das concentrações do Canal e Riacho) e para a saída (concentração no GAV05).

Tabela 5. Relação nitrogênio/fósforo

\begin{tabular}{cccc}
\hline \multirow{2}{*}{ Mês } & \multicolumn{3}{c}{ Ponto } \\
\cline { 2 - 4 } Jan & no interior do açude & de entrada & de saída \\
Fev & 2,093 & 2,277 & 2,173 \\
Mar & 2,568 & 2,391 & 3,050 \\
Abr & 4,986 & 5,911 & 7,250 \\
Mai & 5,906 & 6,043 & 6,240 \\
\hline
\end{tabular}

Conforme a Tabela 5, para todos os meses de estudo a limitação sempre foi da fração nitrogenada $(\mathrm{N} / \mathrm{P}<10)$. Para os pontos localizados no interior do açude e para as entradas a razão N/P foi obtida no mês de abril, respectivamente 5,096 e 6,043 e para a saída do açude o maior valor encontrado para a razão N/P foi de 7,250 no mês de março.

Ambientes com razões N/P baixas são indicativos de eutrofização podendo favorecer as florações de cianobactérias já que o crescimento do fitoplâncton passa a ser limitado pela disponibilidade relativa do nitrogênio favorecendo o crescimento de espécies capazes de fixar o nitrogênio atmosférico (Cotovicz Júnior, 2013). Para o açude Gavião Braga (2006) obteve razões para as concentrações médias de nitrogênio e fósforo de 4,48 (período chuvoso) e 8,55 (período seco) e Freire (2007) encontrou razões para N/P $<<8$ concluindo também que o crescimento fitoplanctônico estava sendo controlado pelo nitrogênio reforçando os resultados aqui obtidos.

Outros estudos verificaram a dominância de cianobactérias em baixas razões N:P, próximos a 15:1 (Havens et al., 2003). Pode haver, ainda, diferenças dentro de uma mesma classe, como é o caso das cianobactérias. Cianobactérias não-fixadoras de $\mathrm{N}$ parecem dominar ambientes com alta razão N:P, enquanto que as espécies fixadoras dominam ambientes que sejam limitados por este nutriente, ou seja, ambientes com baixa razão N:P = 10:1 (Abell et al., 2010).

\section{Conclusões}

1. Foi possível quantificar as cargas dos nutrientes relacionando-se a concentração das espécies químicas com as vazões do açude obtendo-se, para a maioria dos casos analisados, retenção de nitrogênio $(121,42 \mathrm{t})$ e de fósforo $(28,06 \mathrm{t})$ durante o período de amostragem. A única campanha que apresentou exportação de nutriente foi em fevereiro, em que o açude exportou 3,939 $t$ de nitrogênio na forma dissolvida ou gasosa. A partir da quantificação de cargas poluidoras afluentes pode- 
se avaliar o impacto da poluição local e, consequentemente, elaborar medidas de controle eficazes a fim de conservar a qualidade da água do manancial.

2. A determinação da relação Nitrogênio/Fósforo (N/P) permitiu investigar o nutriente limitante do açude; a limitação sempre foi da fração nitrogenada com razões menores que 10:1 $(\mathrm{N} / \mathrm{P}<10)$, sendo este mais um indicador da eutrofização do açude Gavião e passível de favorecer florações de cianobactérias já que o crescimento do fitoplâncton passa a ser limitado pela disponibilidade relativa do nitrogênio, e favorece o aparecimento de espécies capazes de fixar o nitrogênio atmosférico.

\section{Literatura Citada}

Abell, J. M.; Özkundakci, D.; Hamilton, D. P. Nitrogen and phosphorus limitation of phytoplankton growth in New Zealand lakes: implications for eutrophication control. Ecosystems, v.13, p.966-977, 2010.

APHA - American Public Health Association. Standard methods for the examination of water and wastewater. 21.ed. Washington: APHA, 2005. 1207p.

Bennion, H.; Hilton, J.; Hughes, M.; Clark, J.; Hornby, D.; Fozzard, I.; Phillips, G.; Reynolds, C. The use of a GIS-based inventory to provide a national assessment of standing waters at risk from eutrophication in Great Britain. Science and the Total Environment, v.344, p.259-273, 2005.

Braga, E. de A. S. Determinação dos compostos inorgânicos nitrogenados (amônia, nitrito e nitrato) e fósforo total, na água do açude gavião, e sua contribuição para a eutrofização. Engenharia Hidráulica e Ambiental, Fortaleza: UFC, 2006. 120p. Dissertação Mestrado

COGERH - Companhia de Gestão dos Recursos Hídricos. <www.cogerh.gov.ce.br> 4 Abr. 2011.

Conley D. J.; Paerl, H. W.; Howarth, R. W.; Boesch, D. F.; Seitzinger, S. P.; Havens, K. E.; Lancelot, C.; Likens, G. E. Controlling eutrophication: nitrogen and phosphorus. Science Magazine, v.323, p.1014-1015, 2009.

Correll, D. L. Phosphorus: A rate limiting nutrient in surface waters. Poultry Science, v.78, p.674-682, 1999.

Cotovicz Júnior, L. C., Brandini, N., Knoppers, B. A., Mizerkowski, B. D., Sterza, J. M., Ovalle, A. R. C., Medeiros, P. R. P. Assessment of the trophic status of four coastal lagoons and one estuarine delta, eastern Brazil. Environmental Monitoring and Assessment, v.185, p.3297-3311, 2013.

Duarte, A. A. L. S; Vieira, J. P. Integrated estuarial modelling for eutrophication vulnerability assessment. Journal on Fluid Mechanics, v.4, p.1-1, 2009.

Esteves, F. A. Fundamentos de limnologia. Rio de Janeiro: Interciência /FINEP, 2011. 790p.

Figueirêdo, M. C. B. de; Teixeira, A. S.; Araújo, L. de F. P.; Rosa, M. F.; Paulino, W. D.; Mota, S.; Araújo, J. C. Avaliação da vulnerabilidade ambiental de reservatórios à eutrofização. Revista de Engenharia Sanitária e Ambiental, v.12, p.399409, 2007.
Freire, R.; Bonifácio, C. M.; Freitas, F. H. de; Schneider, R. M.; Tavares, C. R. G. Nitrogen forms and total phosphorus in water courses: A study at Maringá stream, Paraná State. Acta Scientiarum: Technology, v.3, p.711-716, 2013.

Freire, R. H. F. Contribuição ao conhecimento limnológico de reservatórios do semiárido brasileiro que abastecem a Região Metropolitana de Fortaleza: Açudes Pacajus e Gavião. Escola de Engenharia de São Carlos, São Carlos: USP, 2007. 246p. Tese Doutorado

Gorayeb, A.; Rosa, M. de F.; Silva, E. V. da; Araújo, L. F. P.; Souza, M. J. N.; Rosa, M. F.; Figueirêdo, M. C. B. Saneamento básico e impactos ambientais na bacia hidrográfica do Rio Curu, Ceará, Brasil. Scripta Nova, v.10, p.10-17, 2006.

Green, M. B.; Finlay. J. C. Patterns of hydrologic control over stream water total nitrogen to total phosphorus ratios. Biogeochemistry, v.99, p.15-30, 2010.

Havens, K. E.; Thomas James, R.; East, T. L.; Smith, V. H. N:P ratios, light limitation, and cyanobacterial dominance in a subtropical lake impacted by non-point source nutrient pollution. Environmental Pollution, v.122, p.379-390, 2003.

Hijo, C. A. G. Quantificação do efeito do açude Castanhão sobre o fluxo fluvial de material particulado em suspensão e nutrientes para o estuário do rio Jaguaribe, Ceará - Brasil. Fortaleza: UFC, 2009. 59p. Dissertação Mestrado

Machado, E. C. M. N.; Galvão, C. O. ; Souza Filho, F. A. Alocação quali-quantitativa da alocação de água em bacias hidrográficas: Metologia multiobjetiva inserida no contexto de recursos hídricos. Revista Brasileira de Recursos Hídricos, v.17, p.213-228, 2012.

Oliveira, T. M. B. F.; Di Souza, L.; Castro, S. S. L. de; Dinâmica da série nitrogenada nas águas da Bacia Hidrográfica Apodi/Mossoró - RN - Brasil. Eclética Química, v.34, p.17-26, 2009.

Pinto-Coelho, R. M.; Giani, A.; Morais-Jr., C. A.; Carvalho-Jr., E. R.; Bezerra-Neto, J. F. The nutritional status of zooplankton in a tropical reservoir: Food quality and community structure. Brazilian Journal of Biology, v.65, p.1-13, 2005.

Rabalais, N. N., Turner, R. E., Diaz, R. J., Justic, D. Global change and eutrophication of coastal waters. ICES Journal of Marine Science, v.66, p.1528-1537, 2009.

Sampaio, E. V.; Rocha, O.; Tundisi, T. M.; Tundisi, J. Composition and abundance of zooplankton in the limnetic zone of seven reservoirs of the Paranapanema river, Brazilian Journal of Biology, v.62, p.525-545, 2002.

Souza Filho, F. de A.; Martins, E. S. R. M.; Porto, M. O processo de mistura em reservatórios do semiárido e sua implicação na qualidade da água. Revista Brasileira de Recursos Hídricos, v.11, p.109-119, 2006.

Vollenweider, R. A. Avaliação do balanço de massa. In: Jørgensen, S. E.; Vollenweider, R. A. Diretrizes para o gerenciamento de lagos. São Carlos: ILEC, IEE, UNEP, v.1, p.43-57, 2000.

Xu, H.; Paerl, H. W.; Qin, B.; Zhu, G.; Gao, G. Nitrogen and phosphorus inputs control phytoplankton growth in eutrophic Lake Taihu, China. Limnology and Oceanography, v.55, p.420-443, 2010. 